\title{
The emerging of the fifth malaria parasite (Plasmodium knowlesi). A public health concern?
}

\begin{abstract}
After examining the most recent scientific evidences, which assessed the role of some malaria plasmodia that have monkeys as natural reservoirs, the authors focus their attention on Plasmodium knowlesi. The infective foci attributable to this last Plasmodium species have been identified during the last decade in Malaysia, in particular in the states of Sarawak and Sabah (Malaysian Borneo), and in the Pahang region (peninsular Malaysia). The significant relevance of molecular biology assays (polymerase chain reaction, or PCR, performed with specific primers for P. knowlesi), is underlined, since the traditional microscopic examination does not offer distinguishing features, especially when the differential diagnosis with Plasmodium malariae is of concern. Furthermore, Plasmodium knowlesi disease may be responsible of fatal cases, since its clinical presentation and course is more severe compared with those caused by $P$. malariae, paralleling a more elevated parasitemia. The most effective mosquito vector is represented by Anopheles latens; this mosquito is a parasite of both humans and monkeys. Among primates, the natural hosts are Macaca fascicularis, M. nemestina, $M$. inus, and Saimiri scirea. When remarking the possible severe evolution of $P$. knowlesi malaria, we underline the importance of an early recognition and a timely management, especially in patients who have their first onset in Western Hospitals, after journeys in Southeast Asian countries, and eventually participated in trekking excursions in the tropical forest. When malaria-like signs and symptoms are present, a timely diagnosis and treatment become crucial. In the light of its emerging epidemiological features, $P$. knowlesi may be added to the reknown human malaria parasites, whith includes $P$. vivax, $P$. ovale, $P$. malariae, and $P$. falciparum, as the fifth potential ethiologic agent of human malaria. Over the next few years, it will be mandatory to support an adequate surveillance and epidemiological network. In parallel with epidemiological and health care policy studies, also an accurate appraisal of the clinical features of $P$. knowlesi-affected patients will be strongly needed, since some preliminary experiences seem to show an increased disease severity, associated with increased parasitemia, in parallel with the progressive increase of inter-human infectious passages of this emerging Plasmodium.
\end{abstract}

Keywords: malaria parasites, Plasmodium knowlesi, emerging plasmodia, human health, public health concerns.

[Braz J Infect Dis 2010;14(3):299-309] @Elsevier Editora Ltda.

During the evolution of the genus Homo (considering the species Homo habilis, Homo erectus, and Homo sapiens), malaria infection accompanied its evolution course, playing a remarkably interesting biological role, and also conditioning its anthropological development. Four main malaria plasmodia involved human beings, although they presented two different evolutionary pathways, from a biological and a philogenetic point of view. In particular, Plasmodium vivax, Plasmodium malariae and Plasmodium ovale had an older evolution which paralleled the human species evolution (the so-called co-evolution), or alternatively they may be "encountered" during the more ancient phases of the development of the genus Homo. Differently, Plasmodium falciparum has been encountered by humans later during their evolution, since it was acquired by monkeys probably between the end of Mesolithic and the beginning of the Neolithic eras. ${ }^{5}$ When considering the role of Plasmodium falciparum, its common and more recent ancestor has been dated at around 100,000 years ago. Moreover,
Authors

Sergio Sabbatani, MD

Sirio Fiorino, $\mathrm{MD}^{2}$

Roberto Manfredi, $\mathrm{MD}^{3}$

${ }^{1}$ Infectious Diseases,

University of Bologna, S.

Orsola-Malpighi Hospital Bologna, Italy.

${ }^{2}$ Internal Medicine, Budrio General Hospital, Budrio, Italy.

${ }^{3}$ Infectious Diseases,

University of Bologna, S. Orsola-Malpighi Hospital, Bologna, Italy.

Submitted on: 06/03/2009 Approved on: 07/19/2009

Correspondence to: Dr. Sergio Sabbatani, MD Infectious Diseases, S. Orsola Hospital Via Massarenti 11 I-40138 Bologna, Italy

Phone: +39-051-6363355

Telefax: +39-051-343500

E-mail: Sergio.sabbatani@ aosp.bo.it

We declare no conflict of interest. 
molecular biology investigations demonstrated a major expansion of this last Plasmodium species in central-eastern Africa only during the last 10,000 years. Such emerging of Plasmodium falciparum has been related to the modification of human habitat, which occurred in that geographical context thanks to the progressive introduction of agriculture and animal breeding. ${ }^{6}$ In nature, non-human primates may become infected by intra-erythrocyte protozoa belonging to the genus Plasmodium, which are different from those that usually sustain malaria infection in humans. Over twenty species of malaria plasmodia are known to be capable of infecting monkeys (Table 1), but the anecdotal transmission of some of these organisms to humans has been considered until recently a rare event with minor public health concerns. ${ }^{7}$ The transmission of these plasmodia protozoa predominantly occurred in Southeast Asia, China, and Central South America. Diagnostic difficulties are also present to confirm these last infections, since the large majority of species that have a simian reservoir are very difficult to be distinguished from the four classical human species, on the ground of the sole microscopical observation (when this last examination still represented the golden standard for the laboratory diagnosis of malaria). ${ }^{8-11}$ The expansion of human activities in geographical areas that remained poorly anthropized in the past (i.e., forest areas where productive activities have been recently started) and wild regions, which are increasingly visited by travellers practicing trekking activities, represent objective elements that lead to an expected increase of risk to acquired malaria plasmodia of simian origin. Moreover, the present demographic expansion of local populations in different regions of the South Asia and South America, which also involved wild areas near to rain forests, especially when villages living on agricultural or breeding activities are exerted, bear further epidemiological risk factors. In these environmental contexts, an overlapping of human and simian habitat becomes evident, in presence of a common infestation by mosquitoes of the genus Anopheles, which are the needed vehicle for malaria transmission to humans. Among the twenty plasmodia species that may infect monkeys, five of them have been documented as potential infectious agents for humans: they are Plasmodium simium, Plasmodium brasilianum (South America), Plasmodium cynomolgi, Plasmodium inui, and Plasmodium knowlesi (Southeast Asia). ${ }^{7}$
Most malaria infection of monkeys tends to cause a mild to moderate disease in humans, which is frequently self-limiting, and infrequently require an antimalarial chemotherapy, since they are often missed or neglected.

In the year $1960^{*}$ the transmission of P. cynomolgi (variety Bastianelli) was reported on the Journal Science in two men exposed to bites of the mosquito Anopheles freebor$n i{ }^{12}$ The infection was documented to come from monkeys (Macachi rhesus). The disease was acquired by laboratory technicians exposed to monkeys, who were in contact with monkeys, during a period in which the animals were repeatedly exposed to sporozoite inoculations. These two patients developed a distinguished fever, which has the typical progression of tertian fever after a period of remission; the incubation time was around 8 days. Subsequently, two voluntary subjects received $10 \mathrm{~mL}$ of blood drawn from one of the two affected patients, and the hypothesis was confirmed, since both men reported malaria attacks, but none of them had an elevated parasitemia. At microscopy, the infecting plasmodia had morphologic features similar to those of $P$. vivax with enlarged red blood cells, which also showed the typical Schuffner's dots in their cytoplasm. The chloroquine treatment prompted a satisfactory response, although one patient suffered of severe malaria paroxysms a few hours after treatment initiation.

These investigators confirmed their earlier observation, thanks to the personal co-operation of another member of the staff who allowed himself to be bitten by an entire swarm of Anopheles freeborni and, after 11-12 days, suffered from headache, general malaise and hyperpyrexia. Since the presence of parasites could not be demonstrated, an indirect method was approached: $10 \mathrm{ml}$ of whole blood of the experimentally infected man were injected to a macaques, and the animal turned out massively infected six days later. The same in vivo experiment was replicated in another staff member and this voluntary also showed the typical malaria paroxysms 14 days after the experimental infection. ${ }^{12}$ Prior to the observation published in the year 1960, regarding the transmission of P. cynomolgi, already in the year 1932, Knoweles and Das Guptu reported the transmission of a Plasmodium of simian origin. ${ }^{13}$ This report was subsequently confirmed through adequate laboratory experiments, and the relevant Plasmodium, which caused daily malaria paroxysms, showing also a morphologic aspect more similar to that of $P$. ma-
*Subsequently, on the basis of studies by Contacos PG et al. (1962), the sub-variety named "Bastianelli" was excluded, so that in subsequent papers only the denomination P. knowlesi remained.

During subsequent years, this malaria infection was artificially induced as a therapeutic agent inducing hyperpyrexia in patients affected by neurosyphilis. ${ }^{10}$

After the inoculation of two voluntaries with infected vec- tors (Anophels stephensi and Anopheles maculatus) and after the inoculation of human parasited blood in five more voluntaries, the published report allowed demonstrating that the presymptomatic period of infections induced by these sporozoites ranged from 31 and 56 days, with parasitemia becoming evident between 21 and 24 days. The artificially induced infections were symptomatic (characterized by fever and chills), and lasted 10 to 26 days. ${ }^{15}$ 
Table 1. Malaria species of simian origin isolated in Asia and in South America (modified from the reference quotation). ${ }^{7}$ Their associated geographical distribution and morphological similarities to one of the four "classical" human plasmodia species (i.e., Plasmodium falciparum, Plasmodium vivax, Plasmodium ovale, and Plasmodium malariae) are pointed out

\begin{tabular}{|c|c|c|}
\hline $\begin{array}{l}\text { Simian } \\
\text { Plasmodium } \\
\text { species }\end{array}$ & Regional distribuition & $\begin{array}{l}\text { Human species } \\
\text { resembling to them }\end{array}$ \\
\hline \multicolumn{3}{|l|}{ Asia } \\
\hline P. coatney & Malaysia, Philippines & P. falciparum \\
\hline P. cynomolgi & $\begin{array}{l}\text { India, Indonesia, Malaysia, Sri Lanka, } \\
\text { Taiwan }\end{array}$ & P. vivax \\
\hline P. eylesi & Malaysia & P. vivax \\
\hline P. fieldi & Malaysia & P. ovale \\
\hline P. fragile & India, Sri Lanka & P. falciparum \\
\hline P. hylobati & Indonesia & P. vivax \\
\hline P. inui & $\begin{array}{l}\text { India, Indonesia, Malaysia, Philippines, } \\
\text { Sri Lanka, Taiwan }\end{array}$ & P. malariae \\
\hline P. jeffrey & Indonesia, Malaysia & P. vivax \\
\hline \multirow[t]{2}{*}{ P. knowlesi } & China, Indonesia, Malaysia, Philippines, & P. malariae, $P$. falciparum \\
\hline & Singapore, Thailand, Taiwan & \\
\hline P. pitheci & Malaysia & $P$. vivax \\
\hline P. simiovale & Sri Lanka & P. ovale \\
\hline P. silvaticum & Malaysia & P. vivax \\
\hline P. youngi & Malaysia & P. vivax \\
\hline \multicolumn{3}{|l|}{ South America } \\
\hline P. brasilianum & $\begin{array}{l}\text { Brazil, Colombia, Mexico, Panama, } \\
\text { Peru, Venezuela }\end{array}$ & P. malariae \\
\hline P. simium & Brazil & P. vivax \\
\hline
\end{tabular}

lariae compared with that of $P$. vivax, was name $P$. knowlesi, in honour of one of the two scientists who discovered it. During the 60s of last century, also another Plasmodium species, named $P$. brasilianum and $P$. inui, which were already recognized as agents of quartan malaria in monkeys, respectively in the New and in the Old World, may parasite humans through mosquito bites. ${ }^{11}$ Also thanks to these last studies, scientific evidences that supported the definitive identification of simian malaria as a zoonosis were reported. ${ }^{10}$

In the year 1965, a case of naturally acquired P. knowlesi infection was described for the first time in a Caucasian man travelling in Malaysia. ${ }^{16}$ This parasite was shown to be easily transmissible to humans through blood inoculation, and was defined by the authors as the human strain $(\mathrm{H}-$ as per "human" species) of P. knowlesi. The work by Chin et al. demonstrated that human infection was characterized by an asexual cycle of around 24 hours, associated with a pattern of daily fever around $104.8^{\circ} \mathrm{F}$, and the concurrent presence of an elevated parasitemia. ${ }^{16}$ Two years later, the mosquito Anopheles balabacensis was demonstrated to be able to transmit the relevant parasite among monkeys. ${ }^{17}$

In the year 1968, the same research group which published the first case of natural transmission, enrolling eight volunteers in a prison located in Atlanta (GA, USA), demonstrated the serial passage of $P$. knowlesi to all subjects involved in the experimental study. These prisoners were exposed to A. balabacensis bites after their pre-contamination realized on infected monkeys. ${ }^{18}$ The present, accurate, study 
confirmed the previous observations and unequivocally concluded that $P$. knowlesi may represent a relevant human pathogen, also supporting the hypothesis that this last Plasmodium may be included in a malaria cycle including monkeys-mosquitoes-man. The authors concluded that if men habitually live in areas affected by the natural transmission of $P$. knowlesi, the vectors will be attracted by both humans and monkeys, and the human exposure may occur with a proportionally elevated frequency. ${ }^{18}$

This issue partially confirmed the observations of Ciuca from Romania, carried out in $1955 .{ }^{19}$ According to this research, a progressive increase of human virulence followed the number of passages of infection. In the original experience from Ciuca, after 170 passages of human blood, a chemotherapy became needed in a large proportion of cases and, in some patients, the parasitemia reached extremely elevated levels, up to 500,000 malaria parasites per $\mathrm{mL}$ of blood. ${ }^{* *}$ In all test subjects clinical manifestations, which had a daily periodicity, were characterized by an elevated parasite load, and were burdened by moderate to severe clinical manifestations.

After these earlier studies, dated around forty years ago, the interest in simian plasmodia seemed reduced over time, but the recent increase of a number of $P$. knowlesi infections case reports in Southeast Asian countries shed again a light on this Plasmodium species, by both epidemiologists and clinical practitioners.

Later in our review, the most recent reports of malaria cases induced by $P$. knowlesi, which lead N.J. White to write his Editorial published in 2008 in the outstanding Journal Clinical Infectious Diseases, to propose this last Plasmodium as the true fifth human malaria pathogen, capable of infecting humans with a remarkable epidemiological impact. ${ }^{10}$

In Sarawak (Malayan Borneo Malese), during the fiveyear period from 1998 and 2002 the annual incidence of registered cases of malaria ranged from 2,496 and 3,155 cases, with $P$. vivax as the predominating species $(69.1 \%$ of episodes), and $P$. falciparum presenting a significantly lower frequency (19.7\%). In the same area, the parasites identified as $P$. malariae were $9.4 \%$ only, the mixed forms accounted for $1.8 \%$ of cases, while no episode due to $P$. ovale was identified. P. malariae parasites appeared concentrated in a specific region (Kapit), which is particularly rich in rain forest (Figure 1), and they represented $20 \%$ of all malaria cases but, surprisingly, these plasmodia presented unexpected features at microscopic examination. Moreover, when compared with usual malaria infections, these episodes occurred

$* *$ Chin et al. wrote that their serial inoculations were not sufficient in number to confirm such conclusions, although it is interesting to notice that in the second group of volunteers, who received sporozoite inoculations, the maximum parasitemia tested significantly more elevated $(3,450$ to $6,950 / \mu \mathrm{L})$, when compared with extremely elevated blood parasite loads (between 48 and 66,640 parasites per $\mu \mathrm{L}$ of blood); finally, these patients needed a systematic treatment and hospitalization at the Kapit hospital, due to severe signs and symptoms and the relevant clinical features. ${ }^{8}$ These atypical features led the same researchers to perform a preliminary study with a nested PCR malaria detection assay in five isolates microscopically identified as $P$. malariae: surprisingly, plasmodial species DNA was demonstrated, but the search tested negative also for all the four major human plasmodial species, including P. malariae. ${ }^{8}$ This preliminary experience induced the same researchers to perform studies on a more extended sample of patients with malaria. Between March 2000 and November 2002, blood specimens from 208 persons who acquired confirmed malaria in the Kapit region were collected. Molecular biology assays were applied on these clinical samples; in particular, for eight samples, initially identified as containing $P$. malariae strains by conventional microscopy, the small subunit ribosomal RNA (SSU rRNA) and the circumsporozoite protein (csp) genes were sequenced. These blood specimens were investigated with a gene-specific (speciesspecific) nested PCR, also using recently prepared primers, which were specific for P. knowlesi. The results proved surprising, since all plasmodial DNA sequences were philogenetically not different from those of $P$. knowlesi, and also proved significantly different from those of other known human malaria parasites. In particular, 58 of 208 patients affected by malaria proved positive for a $P$. knowlesi infection. In these episodes, the parasitized erythrocytes were morphologically indistinguishable from those parasitized by $P$. malariae; furthermore, the majority of these cases regarded adult subjects. ${ }^{8}$ After this work, the same study group published a more extensive research, carried out on the following sample including: i) 960 patients with malaria, hospitalized in Sarawak between years 2001 and 2006; ii) 54 hematological archives, classified as patients with a probable P. malariae infection, coming from 15 different districts of the Malaysian State of Sabah, collected between 2003 and 2005, and four districts of the Pahang region (peninsular Malaysia), collected between 2004 and 2005; iii) four patients whose cause of death was a suspected P. knowlesi malaria.

The molecular biology assay, performed by a nested PCR technique, demonstrated that $P$. knowlesi DNA has been found in $27.7 \%$ of the 960 patients of the first study sample, in $83.7 \%$ of subjects coming from Sabah region (second patient sample), and in all patients recruited from the Pahang

with values ranging from 850 and $1,600 / \mu \mathrm{L}$ of the first group of subjects receiving inoculations. Moreover, the first two induced infections were self-limited, while a chemotherapy intervention became necessary in three out of four volunteers, included in the second study group. ${ }^{18}$ 
Figure 1: Satellite photograph of the city of Kapit geographical area and its surrounding forest. The area is particularly rich in rivers and channels.

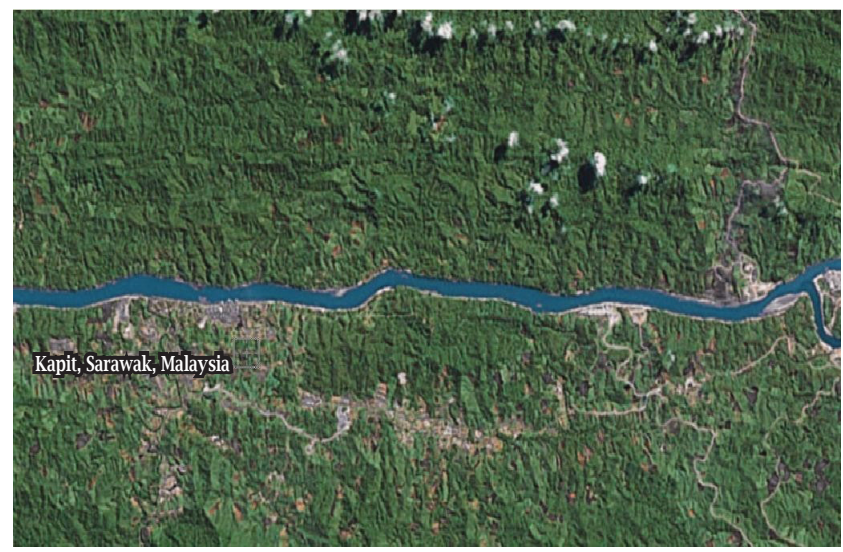

districts. Furthermore, in all the four deceased patients, $P$. knowlesi DNA was found in associated with an elevated parasite load and a remarkable liver-kidney dysfunction. ${ }^{9}$ This last quoted paper definitively demonstrated that P. knowl$e s i$, although in its initial forms, has a strict morphological similarity with $P$. malariae, has a clearly increased clinical impact, and may cause life-threatening diseases.

The different impact is related to the evidence that $P$. knowlesi has a daily replication cycle, and may therefore reach elevated life-threatening levels of parasitemia in absence of an effective treatment, whereas $P$. malariae, as known, has a replication cycle every three days (responsible for the socalled quartan fever), and never reaches so elevated blood parasite concentrations. As anticipated in the initial investigation, the patients infected with $P$. malariae never attained a parasitemia above 5,000 involved erythrocytes per blood $\mu \mathrm{L} .{ }^{9}$ On the other hand, P. knowlesi interestingly, although not significantly segregated in the microcirculation, when achieving an elevated parasitemia becomes rapidly lethal in Rhesus monkeys (Macaca mulatta). ${ }^{10}$

In the Editorial written by N.J. White for the Journal Clinical Infectious Diseases, the author focuses on the four deceased patients, underlying their proportionally advanced age (range 39-69 years) and the constant report of abdominal pain and fever. In two cases with elevated parasitemia an evident anemia was found, while all reported subjects showed a deep thrombocytopenia (below 30,000 cells/ $\mu \mathrm{L}$ ), kidney and liver failure with frank jaundice (which are typical features of severe $P$. falciparum malaria); a leukocytosis was found in three of four cases. ${ }^{10}$

Historical studies performed in the sixties on the microcirculation of Rhesus monkeys infected by malaria plasmodia detected the presence of red blood cells in capillaries and small veins; based on these evidences, N.J. White discusses the similarity of the severe human disease compared with that of monkeys, and in particular underlines the need to assess the relationship between the overall parasite load and disease severity. ${ }^{10}$ After excluding a concurrent sepsis (as a possible cause of the metabolic acidosis observed during severe malaria, N.J. White recommends the administration of antimalarial drugs since the early disease occurrence. ${ }^{10}$ Finally, concluding his Editorial, the author encourages extreme attention when facing Asian cases of malaria characterized by elevated parasitemia at microscopy (which remains the diagnostic standard until now), in order to prevent a complicated or fatal course. ${ }^{10}$

At this point, coming back to the title of our review, we are concerned about the possibility that $P$. knowlesi may represent a public health problem. Multiple, different conditions have to be concurrent to support the transmission of simian plasmodia to humans. First of all, the human erythrocytes must be susceptible to the invasion by simian plasmodia; secondarily, the environmental proximity of rain forests where infected monkeys are present; finally, mosquitoes belonging to the genus Anopheles, able to bite both humans and monkeys, must be present, and actively perform their vector function.

After the report of numerous cases of $P$. knowlesi infection during the last decade, it became relevant to definitely establish which have been the vectors responsible for transmission and to clarify all the dynamics of the transmission of this last Plasmodium species to human kind.

In the Malaysian peninsula, simian plasmodia were reported for the first time in the year $1908,{ }^{20}$ but the experiments carried out in the 1960s, which showed that P. cynomolgi might be transmitted to humans through mosquito bites, ${ }^{12}$ promoted an increased attention from scientists, until now.

Vythiliam et al. recently published an entomologic study, which contributed to clarify many open questions. ${ }^{21}$ In a period of five months, mosquitoes were captured in three different ecological sites: a "longhouse", a typical Malaysian house (Figure 2), a farm located at the edge of

Figure 2: Microbiological (hemoscopic) examination of peripheral blood, which shows an elevated number of erythrocytes parasitized by $P$. knowlesi.

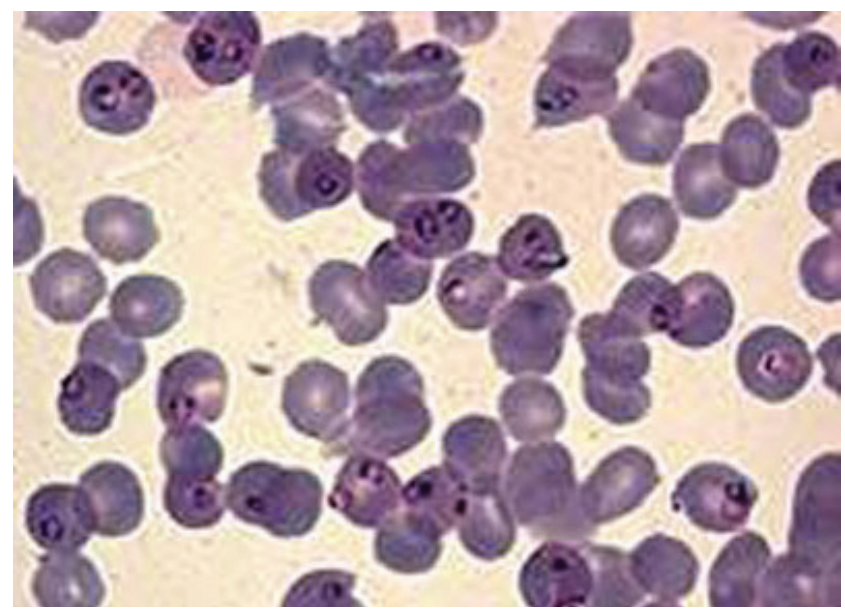


rain forest, and in the forest itself. The capture of insects was operated after Anopheles mosquitoes have been attracted by humans and monkeys; after the identification of sporozoites and oocysts in the selected mosquitoes, these biological specimens were preserved in $100 \%$ alcohol, and subsequently plasmodial DNA has been extracted, and samples have been analyzed by a nested PCR assay. Nine different species of Anopheles species have been identified, with Anopheles latens as the predominant one (50.5\% of cases). This last mosquito species was formerly named Anopheles leucosphyrus, which was known to attack both humans and monkeys (i.e., Macaca fascicularis). These monkeys are affected by mosquito bites especially when they are located over the trees, three to six meters over the ground. In this study, four A. latens mosquitoes tested positive for plasmodial sporozoites, and four for plasmodial oocysts, with a parasitemia rate of $1.18 \%$. All these insects had been captured while they bite humans, and they proved positive in the search for $P$. knownlesi DNA, based on a nested PCR assay. A. latens mosquitoes have been found in farm and also at the edge of forest, but they were not present in central areas of rain forest. These insect attacked humans from $6 \mathrm{pm}$ and overnight, with a maximum peak around midnight. The rate of insect bites between humans and monkeys was 1:1.12, when considering all $A$. latens female insects. The bite rate of

$* \cdots *$ The Kapit district is a hilly area covered by tropical forests. The traditional house of natives is represented by the longhouse (Figure 3 and Figure 4), and mosquitoes were captured in one of these houses, surrounded by trees and bushes, in the countryside named Lubok Loh Young, which us located 16 $\mathrm{km}$ far from the town centre and close to a river. The second investigated site was a factory at Ulu Slugai Young, located in a deforested site which was converted to an area cultivated with fruit trees, but was subsequently surrounded by the primary forest, and was "attacked" by wild tropical vegetation. A small cabin with a close, small river are also part of this region, while the only existing house is located immediately

Figure 3: A typical Malaysian longhouse is represented.

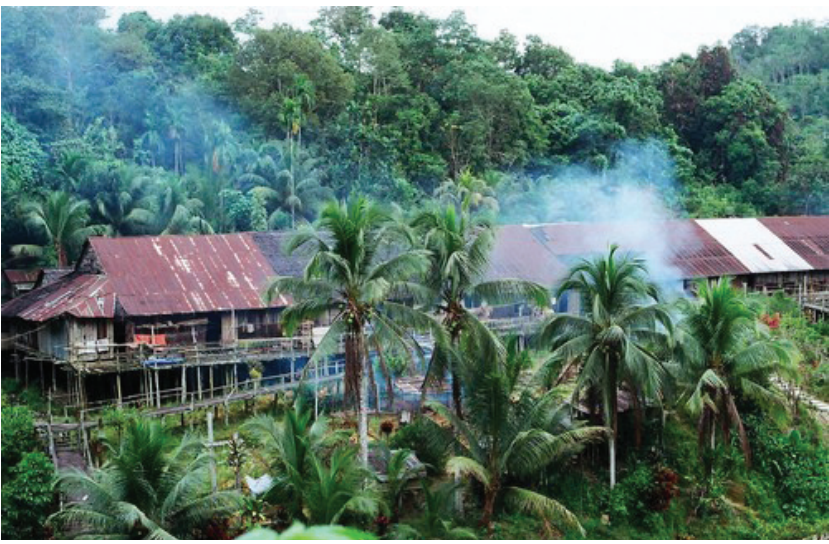

humans by A. latens was more elevated at the forest limits $(6.74 \%)$, while lower rates were found in the rain forest $(1.85 \%)$ and in the examined longhouses $(0.28 \%))^{21}$ Previous historical studies performed nearly 50 years ago, identified $A$. hacheri as a possible vector of $P$. knowlesi, ${ }^{22}$ but further information excluded that this last mosquito is attracted by humans, while it bites monkeys in their natural environment. The judgement of entomologists is that man may acquire infection in the forest during hunting or returning to the farm at sunset, after the working day. When considering the vector, it acquires the infection from wild monkeys: this opinion is enforced by the absence of epidemic clusters inside the local longhouses, and the fact that the major number of infected mosquitoes has been registered in the forest, or close to the forest edge. $^{21}$

In the year 2008, the same researchers published the results of another entomological investigation conducted in the Kapit ${ }^{* *}$ district of Sarawak (Malaysian Borneo). ${ }^{23}$ Also in this last study, mosquitoes have been captured in three different sites: the tropical forest, a factory close to the forest edge, and a longhouse. The salivary glands and the digestive tract of these mosquitoes were examined, to establish the eventual presence of malaria parasites. In the time range of 11 months, from June 2005 up to April 2006, 2,504 mosquitoes of the genus Anopheles were col-

outside the farm. Pig-tail macaques are frequently observed in this region, and one of the farm inhabitants was hospitalized at Kapit Hospital due to a P. knowlesi malaria attack; the distance between the longhouse and the farm is around $5 \mathrm{~km}$. The third examined site is located into the rain forest, $4.5 \mathrm{~km}$ far from the Kapit town centre, at an altitude of 1,200 meters over the sea. This forest area has road accessible to equipped motor vehicles, save during the rainy season, when the only access feasible is by foot. Long-tail macaques have been frequently observed in this site, which is located at around one $\mathrm{km}$ far from the house of patients hospitalized in 2001 for malaria attack. ${ }^{23}$

Figure 4: The interior features of a longhouse.

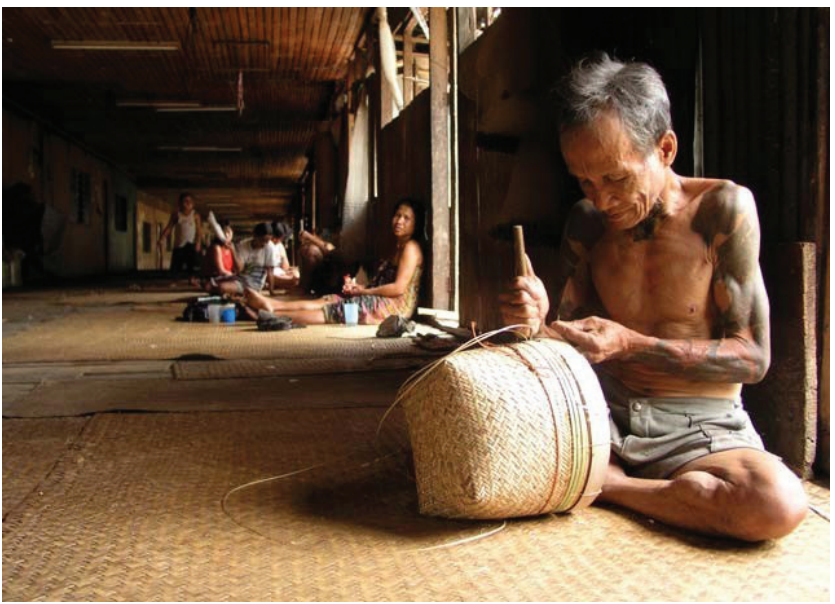


lected, which belonged to 12 different species: 1,035 were from the farm, 774 from the forest, and the remaining 425 from the longhouse. In the rain forest, the two predominant species were A. latens (62.3\%) and l'A. watsoni (30.6\%), while in the farm A. donali prevailed (49.9\% of cases), followed by $A$. latens (35.6\%); in the longhouse, $A$. latens (29.7\%), A. donaldi (22.8\%), and A. Vanus (21.9\%) represented the most common retrieved mosquito species. A. latens was the only mosquito species that tested positive at the sporozoite search, and this species resulted able to attack both monkey and humans. The researchers observed that monkey were attack both on three and at the ground level, and the peak time of bites of $A$. latens was around $6 \mathrm{pm}$ in the evening. ${ }^{23}$ Since the 1960s, P. knowlesi was demonstrated to represent the first plasmodial species which developed an antigenic variation. ${ }^{24}$ In 1983, a paper was published which demonstrated that this last Plasmodium species had a strict filogenetic relationship with $P$. vivax, ${ }^{25}$ but despite these correlations, remarkable phenotypic differences remained, including the preference for host blood cells, the absence of an hepatic latency (hypnozoites), and the length of the asexual cycle, ${ }^{25}$ in the case of P. knowlesi. ${ }^{25}$ Recently, in 2008, the Journal Nature published the analysis of the nuclear genomic sequencing of $P$. knowlesi. It was the first complete gene sequencing of a simian Plasmodium, therefore offering the opportunity for a comparison with the complete genome of $P$. vivax and other already known plasmodia genomes. ${ }^{25}$ In contrast with other plasmodial genomes, putative familiar antigenic variants are scattered throughout the genome and associated with intrachromosomal telomere repeats. One of these families, called KIRs, includes genomic sequences which on the whole match around one-half of the extracellular domain CD99 of the host: this situation suggested that this form could represent an unusual molecular mimicry. ${ }^{26}$

P. knowlesi-related malaria, after the epidemic foci reported in the Malaysian peninsula (Pahang), in Sarawak (the first Malaysian state located in the great Borneo island), and in the Sabah territory (the second Malaysian state, located in the Northern portion of the big island), has been extensively studied, according to a broad range of research features.

This growing attention of researchers and international health care authorities led to an increase of awareness and level of attention of practitioners who face potential patients coming from Southeast Asia. ${ }^{7}$ In fact, during the past few years and during the first months of 2009, a number of reports regarding cases of travellers hospitalized in Western hospitals after coming from tours in Malaysia have been published. In March 2007, a 53-year-old man from Finland was admitted to a local Hospital after a four-week journey in Peninsular Malaysia. ${ }^{27}$ No antima- larial prophylaxis had been performed. The tour included a couple of weeks spent in the capital city Kuala Lumpur, with excursions of some day in surrounding rural areas. Later, after driving along the coast toward North-Western regions of Peninsular Malaysia, he remained for five days in the rain forest, about $80 \mathrm{~km}$ away from Ipoh. During this time, the traveller slept in a house lacking protection from mosquitoes, and he did not use any repellent. The patient spent his last week of the Malaysia tour in the Langkawi Beach area, where he spent his time in highrank hotels. When still in Malaysia, the patient suffered from occasional abdominal pain, spontaneously recovered after coming back to Finland. Three days after returning back to home, a febrile peak (body temperature $38.8^{\circ} \mathrm{C}$ ) appeared, followed by irregular fever in subsequent days, so that he was referred to a local hospital. Upon admission, laboratory examination showed only a moderate leukopenia, and a peripheral blood sample allowed to make diagnosis of $P$. falciparum infection, with a very low parasitemia $(<1 \%)$. A treatment with quinine IV and oral doxicyclin was immediately started. Owing to the persistence of fever, on the subsequent day the patient was transferred to the University Hospital of Helsinki, where microscopical examinations were repeated, and a suspect of atypical plasmodia emerged, so that the laboratory identified a P. falciparum and P. malariae co-infection. During a 10-day course with quinine plus doxicyclin, the patient suffered from hypoglicemia and a mild and transient visual and auditory impairment, and a mild lymphopenia. At the second day of admission, a blood specimen was sent for molecular biology studies. A first PCR assay performed with the primers "rOval" and "rPLU2" (DNA templates produced in Basel, Switzerland, and purified from $200 \mu \mathrm{L}$ of erythrocytes through the QIAamp DNA Mini Blood Kit, Qiagen, Helsinki, Finland), did not achieve amplification. Other PCR assays were repeated with two alternative primers (rPLUS and rPLUS2), derived from a preserved region of the $18 \mathrm{Sr}$ RNA marker gene, allowed to attain an amplicon.

These results initially lead to suspect a $P$. ovale infection and, according to this hypothesis, a 14-day primaquine course was administered, in order to eradicate eventual liver hypnozoites. The laboratory products of gene amplification were subsequently submitted to a direct nucleotide sequencing, and was found to be identical to two sequences of P. knowlesi; the first one was an human isolate coming from the Malaysian Borneo (AY327556), and the second one a simian sample of a Macaca mulatta coming from Colombia (U72542), both already assessed and stored in the Genbank database. The researchers identified six other published gene sequences of P. knowlesi, which were different compared with the sequence of the patient from Finland for one nucleotide 
only (99\% identity rate). The comparison of the examined DNA sequence with the $P$. ovale primer showed a similarity limited to $50 \%$ only. ${ }^{27}$ These evidences induced the authors from Finland to identify their Plasmodium as P. knowlesi, imported after travelling in Malaysia. ${ }^{7}$

A second case has been reported from the US: a 50year-old woman born in the Philippines, but living in the US since for 25 years, returned to native country to visit relatives and friends, in October 2008. ${ }^{7}$ When in the Philippines, the patient visited the touristic site of Palawan, remaining for a few days in a cabin located at the edge of a rain forest, usual habitat of long-tail macaques. This patient also never took an antimalarial prophylaxis, and did not use any protection against mosquito bites. After coming back to the US at the end of October 2008, she suffered from headache, associated with recurring chills and hyperpyrexia, which led to hospitalization. Upon admission, the patient had low blood pressure, and laboratory studies detected a thrombocytopenia. Although physicians asked for search of malaria plasmodia, an initial, wrong diagnosis of babesiosis was posed and corrected next day for a diagnosis of malaria, after revising the microscopic aspect of intracellular parasites: the parasitemia was estimated at $2.9 \%$ of erythocytes, but the atypical appearance of protozoa did not allow making a species-specific identification. Anyway, a favourable course followed a timely treatment with atovaquone, proguanil, and primaquine. In order to obtain a specific diagnosis, two blood samples were sent to the Wadsworth Center Parasitology Reference Laboratory, when molecular biology testing was available. At this reference centre, atypical plasmodia were observed, but a conventional PCR assay, which recognizes the small rRNA subunit (SSU), failed in confirming an infection due to one of the four known species of human malaria plasmodia. The sample tested also negative for some variants of $P$. ovale, which may be present in Southeast Asia. Further bio-molecular assays based on primers, which are specific for the SSu rRNA of the genus Plasmodium, allowed to sequence a 1055-bp genome, which proved $99 \%$ similar in its complete length to the SSU rRNA gene of P. knowlesi ( $\mathrm{H}$ strain). ${ }^{7}$ On the ground of these results, $P$. knowlesi was quoted as the fifth human malaria parasite.

Recently, single case reports of native subjects who acquired $P$. knowlesi malaria were recorded from Thailand, ${ }^{28}$ China, ${ }^{29}$ and Singapore. ${ }^{30}$ In all these cases, the diagnosis was confirmed by a specific PCR assay.

During the first months of 2000, the case of a 35-yearold Swedish traveller who made a two-week journey to Sarawak during October 2006 has been published. ${ }^{31}$ During his second week of travel, the patient (who did not take any antimalarial chemoprophylaxis), made a trip through the jungle of Bario Highlands, a territory located between 800 and 1,400 meters over the sea. Signs and symptoms appeared 11 days after leaving Bario. Coming back to Sweden, the patient was admitted at a Stockholm Hospital referring hyperpyrexia (body temperature $40^{\circ}$ C) for two days, sweating, headache, and fatigue. Blood pressure and respiratory frequency proved within normal limits. The screening laboratory testing showed a moderate leukopenia, thrombocytopenia, and elevated plasma levels of C-reactive protein, together with a mild hyperbilirubinemia and a modest alteration of serum transaminases. Since malaria was suspected, notwithstanding a rapid malaria assay that tested negative, a therapy was started with oral mefloquine. This approach was correct, since the microscopic search of plasmodia tested positive, although with a low $(0.1 \%)$ rate of infected erythrocytes. The plasmodial species was not identified, but a $P$. malariae was suspected. The authors recorded that tardive trofozoites were observed, and the parasitized red blood cells did not appear increased in size. Due to an effective treatment, the patient was discharged after two days, with no more fever. At the time of this report, the only available PCR assays in Sweden were those of the four "classical" known human pathogens, so that all molecular biology tests remained negative. When considering the epidemiological and microscopical issues (recent travel to Malaysia, and a doubt microscopic identification of plasmodia), a $P$. knowlesi involvement was suspected. As a consequence, a frozen blood sample was forwarded to the Centre for Malaria Research of the Malaysian University of Sarawak. According to usual techniques, the plasmodial RNA was extracted and identified, and the PCR amplification was successfully obtained. Cloning and sequencing of the A-type small subunit ribosomal (SSUr) RNA gene confirmed the diagnosis of P. knowlesi infection. In addition, phylogenetic analysis of the malaria parasite of Swedish patient was overlapping with that of other isolates of this plasmodial species, which has been previously characterized in Malaysia. ${ }^{31}$

\section{CONCLUDING REMARKS}

After the discovery of P. knowlesi in 1932 and the subsequent demonstration of its possible transmission from monkeys to humans, some attempt to apply an experimental "malaria therapy" to patients affected by severe neurosyphilis were made, actively infecting these neuro-psychiatric patients. ${ }^{10}$ After demonstrating the possible fatal course of $P$. knowlesi transmission, further approaches were realized by injecting $P$. vivax parasites. ${ }^{10}$ Also thanks to these therapeutic experiments of 70 years ago, which now is considered remarkably criticisable according to an ethical perspective, the potential virulence of $P$. knowlesi malaria was recognized and confirmed. 
The observation carried out in 1967, when the transmission of $P$. knowlesi was experimentally demonstrated to occur from monkeys to human via the vector A. balabacensis, allowed to include human $P$. knowlesi disease among zoonosis, bur for some decades only a few reports were recorded, which probably depended on a poor diagnostic sensitivity, more than a true, poor epidemic impact. The epidemiological surveys conducted in Malaysia, which demonstrated that $P$. knowlesi is the major cause of malaria in that geographical context, led to confirm that the disease related to this last plasmodial species plays an important role, and allowed to classify $P$. knowlesi as the fifth causative agent of human malaria. ${ }^{11} P$. knowlesi has a daily replication cycle, and if a timely diagnosis and a rapid and effective treatment are missed or delayed, may attain parasitemic levels which are frankly life-threatening. As anticipated, a relevant problem is related to the cumbersome species identification through plain microscopic examination: during early disease stages, $P$. knowlesi is very similar to P. malariae. This last Plasmodium species has a lower mortality rate, since it replicates every three days, and this plasmodial infection coexist with human kind since immemorial time, and had a prolonged co-evolution phase with human ancestors (like Homo habilis, Homo erectus, and Homo sapiens).

Regarding the virulence of $P$. knowlesi and its possible expansion, we must emphasize that the studies performed in Romania stated that increased human transmissions increase the pathogenicity of these protozoa. According to this last observation, a significant worsening of disease severity could be observed in parallel with the progressive extension of its geographical extension. Relevant, general information descend from researches performed in Malaysia. The human habitat is more and more overlapped to that of some primates, and this is mainly due to two aspects: the first one is represented to the expansion of human activities (agriculture, deforesting, clearing of valued wood, and animal breeding) in areas also inhabited by monkeys. The second emerging issue is related to the relevant increase of touristic activities (i.e., trekking) by international visitors, who spend a proportionally limited time in this environment, and subsequently return to their countries of origin. In this last situation, the limited knowledge of simian plasmodia and their diagnostic and identification problems may confuse Western health care providers, especially when a basic microscopic, parasitological examination is carried out for the research and identification of malaria plasmodia, which does not allow distinguishing P. malariae from P. knowlesi, therefore posing at risk patient's life. The overcoming of this problem has been obtained thanks to molecular biology techniques, and may help solve the difficulties of a timely diagnosis of plasmodial species, although the organizational aspects linked to the availability of PCR assays have to be rapidly solved, especially when Western Hospital where taking care of travellers coming back from endemic tropi- cal countries of Southeast Asia are assessed. The molecular biology techniques have been demonstrated to be extremely useful in describing the general epidemiological features of malaria, and to clarify the role and importance of mixed infections, which still remain largely underestimated. ${ }^{10}$ On the base of epidemiological features of regions where epidemic foci of P. knowlesi malaria have been reported and the anecdotal episodes of clinical reports occurred in Western travellers returning to their countries of origin with a $P$. knowlesi disease, it is not possible to establish whether infections have been acquired by the most common animal reservoirs (i.e., macaques), or whether human-to-human contagion may occur. However, it is presently clear that this zoonosis is no more responsible for single, episodic cases, but it represents a true health care emergency in Southeast Asian countries.

From a clinical-therapeutically point of view, although the majority of affected patients experienced a rapidly favourable response to oral cloroquine and other antimalarial compounds, the four fatal cases of $P$. knowlesi disease were recognized only within seven days from the onset of signs and symptoms. The most frequent clinical features of these subjects included fever, abdominal pain, thrombocytopenia, kidney failure, and jaundice. All patients who deceased in Malaysia received an incorrect diagnosis of $P$. malariae. The recommendation coming from the potential, fatal evolution of these disease episodes is that persons coming from Southeast Asia, manifesting daily fever, and with a microscopic evaluation suspect for $P$. malariae infestation, especially when an elevated parasitemia is present, should be reassessed for a P. knowlesi disease and treated adequately and promptly, to avoid liver-kidney complications. ${ }^{10}$ In all this cases, the resort to PCR assays for a correct molecular biology definition plays an essential role.

In conclusion, a general, speculative consideration may be expressed. The fitting of human kind to the environment never ceased during time, while the evolution of biological factors (i.e., plasmodia, or vectors) steadily proceeded following the environmental modifications, and selecting features which were more favourable to their success. Humans and their ancestors, through genetic, immunological, cultural, and behavioural adaptive processes, succeeded to give responses adequate to successfully overcome the evaluative pressure exerted by these parasites. The oldest plasmodia $(P$. vivax, $P$. ovale, and $P$. malariae), which had a co-evolution with human ancestors, subsequently joined $P$. falciparum at the end of Mesolithic era and at the onset of Neolithic era. In this perspective, we remind that the development of agriculture led to an overlapping of human habitat and that of $P$. falciparum. In Borneo and in Malaysia (in general), the human activities had a rapid growth just in the last 50-60 years at interested regions which were poorly inhabited in previous times, where a close contact with some primate varieties (i.e., Macaca fascicularis), natural hosts of $P$. knowlesi, which 
in presence of an effective vector (A. latens), may infect human with a non-episodic frequency.

Figure 5 allows to appreciate the global co-evolutionary path of malaria plasmodia, in parallel with that of the genus Homo, from hominids (Group A), towards modern men (Group C), with the arising of $P$. falciparum, which entered the epidemiological scenario of infecting human of Mesolithic and early Neolithic eras. These men lived in cabins located at the edge of rain forest and in the proximity of the first cultured fields (Group B): the introduction of the first organized human activities alone have significantly contributed to the expansion of P. falciparum, which so far represents the most relevant malaria pathogen from the pathogenesis of pre-historical populations, inevitably conditioning both economic and anthropological development.
The hope is that epidemic foci reported from Southeast Asia remain circumscribed in that geographical context, and the infection does not spread to other areas, which are susceptible due to similar climatic and environmental features, and for the presence of other possible natural hosts. ${ }^{* * *}$ In this case, the impact of autochthonous populations, not naturally immunized, after contacts that normally occur during early infancy, may be responsible for particularly severe consequences, when considering the mimicry P. malariae has with P. knowlesi. Moreover, we have to underline the early observations by Ciuca, ${ }^{19}$ partially confirmed by Chin et al. ${ }^{18}$ regarding the tendency to a gradual increase of $P$. knowlesi virulence in humans, through the progressive increase of the number of host passages.

Figure 5: The graph depicts the biological and phylogenetic evolutions of P. vivax, P. malariae, and P. ovale (Group A), compared with the extremely more recent evolution pathway of $P$. falciparum (Group B). P. knowlesi (belonging to Group C) is the last Plasmodium species which steadily entered the human pathology, and is related to productive and touristic human activities carried out in the forest regions of Malaysia and Borneo. The image has been taken and modified from some studies published by L. Capasso (quoted among references) ${ }^{1-3}$ specially with Group C malaria parasites.

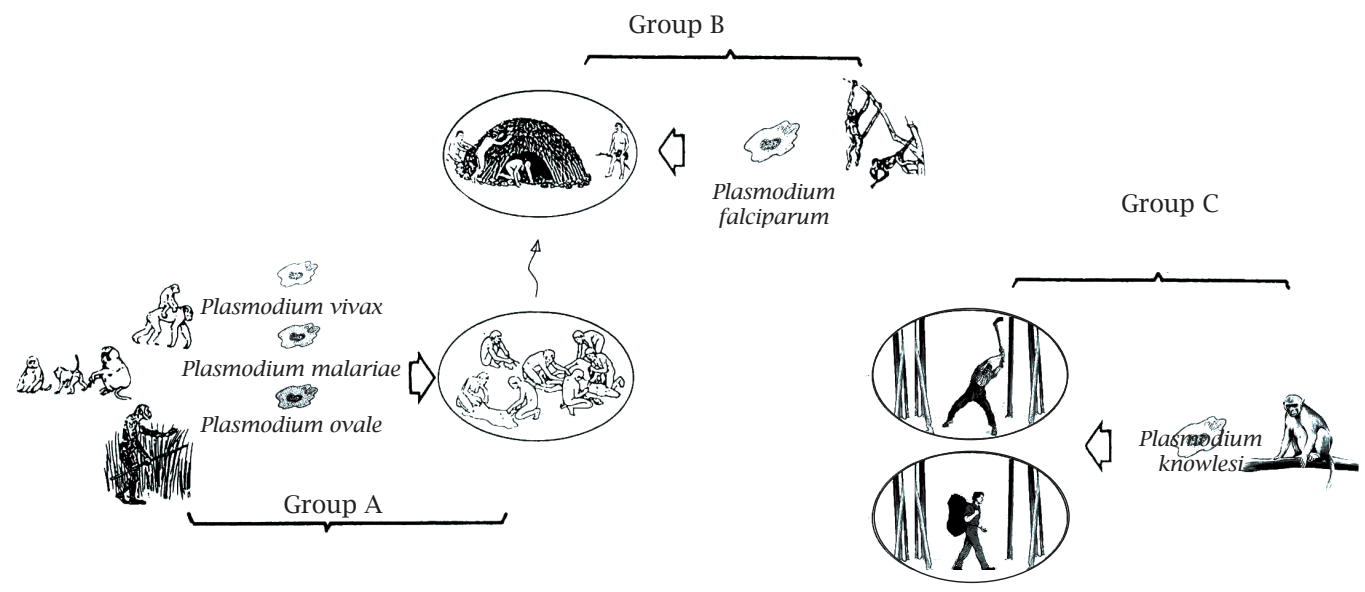

****Noteworthy, besides Macaques Rhesus (M. mulatta), pig-tail monkeys (M. nemestina), long-tail monkey (M. inus), and squirrel monkey (Saimiri sciurea) also received infected blood, and all of them became parasitized. 


\section{REFERENCES}

1. Capasso L. L’origine delle malattie. Chieti, Italy: M. Solfanelli; 1985.

2. Capasso L. Salute e malattie nell'evoluzione umana. Chieti, Italy: M. Solfanelli; 1988.

3. Capasso L, Di Tota G. Paleopatologia delle malattie infettive: importanza evolutiva. In: Peretto C, Millikem S. Atti XI Congresso degli Antropologi Italiani. L'adattamento umano all'ambiente passato e presente; 1995 Sep 13-16; Isernia, Italy; p. 309-22.

4. Cohen MN, Armelagos GJ, editors. Paleopathology at the Origins of Agriculture. Orlando (USA): Academic Press Inc.; 1984.

5. Sallares R, Bouwman A, Anderung C. The Spread of malaria to Southen Europa in Antiquity: New Approaches to Old Problems. Med Hist 2004; 48:311-28.

6. Joy DA, Feng X, Mu J et al. Early origin and recent expansion of Plasmodium falciparum. Science 2003; 300:318-21.

7. Centers for Disease Control and Prevention (CDC). Simian malaria in a US traveler. M.M.W.R. 2009; 58:229-32.

8. Sing B, Sung LK, Matusop A et al. A large focus of naturally acquired Plasmodium knowlesi infections in human beings. Lancet 2004; 363:1017-24.

9. Cox-Singh J, Davis TME, Lee KS et al. Plasmodium knowlesi malaria in humans is widely distributed and potentially lifre threatening. Clin Infect Dis 2008; 46:165-71.

10. White NJ. Plasmodium knowlesi: the fifth human malaria parasite. Editorial Commentary. Clin Infect Dis 2008; 46:172-3.

11. White NJ. Sharing malarias. Lancet 2004; 363:1006.

12. Eyles DE, Coatney GR, Getz ME. Vivax-type malaria parasite of macaques transmissible to man. Science 1960; 131:1812-3.

13. Knowles R, Das Gupta BM. A study of monkey malaria, and its experimental transmission to man. Ind Med Gaz 1932; 67:301-21.

14. Contacos PG, Lunn JS, Coatney GR, Kilpatrick SW, Jones FE. Quartan-type malaria parasite of New World monkeys transmissible to man. Science 1963; 142:676.

15. Coatney GR, Chin W, Contacos PG, King HK. Plasmodium inui, a quartan-type malaria parasite of Old World monkeys transmissible to man. J Parasitol 1966; 52:660-3.

16. Chin W, Contacos PG, Coatney GR, Kimball HR. A naturally acquired quotidian-type malaria in man transferable to monkeys. Science $1965 ; 149: 865$.
17. Collins WE, Contacos PG, Guinn EG. Studies on the transmission of simian malarias. Transmission of the $\mathrm{H}$ strain of Plasmodium knowlesi by Anopheles balabaensis balabacensis. J Parasitol 1967; 53:S41-S44.

18. Chin W, Contacos PG, Collins W, Jeter MH, Alpert E. Experimental mosquito transmission of Plasmodium knowlesi to man and monkey. Am J Trop Med Hyg 1968; 17:355-8.

19. Ciuca M, Lupasco G, Negulici E, Costantinescu P. Research on the experimental transmission of Plasmodium malariae to man. Arch Roum Exp Microbiol 1964; 23:763-76.

20. Daniels CW. Animal parasites in man and some of the lower animals in Malaya. Stud Inst Med Res FMS 1908; 3:1-13.

21. Vythlingam I, Tan CH, Asmad M, Chan ST, Lee KS, Singh B. Natural transmission of Plasmodium knowlesi to humans by Anopheles latens in Sarawak, Malaysia. Trans R Soc Trop Med Hyg 2006; 100:1987-88.

22. Wharton RH, Eyles DE. Anopheles hackeri, a vector of Plasmodium knowlesi in Malaysia. Science 1961; 139:279-80.

23. Tan CH, Vythlingam I, Matusop A, Chan ST, Singh B. Bionomics of Anopheles latens in Kapit, Sarawak, Malaysian Borneo in relation to the transmission of zoonotic simian malaria parasite Plasmodium knowlesi. Malaria J 2008; 7:52-9.

24. Brown KN, Brown IN. Immunity to malaria: antigenic variation in chronic infections of Plasmodium knowlesi. Nature 1965; 208:1286-8.

25. Carlton JM, Adams JH, Slva JC et al. Comparative genomics of the neglected human parasite Plasmodium vivax. Nature 2008; 455:757-63.

26. Pain A, Böhme U, Berry AE et al. The genome of the simian and human malaria parasite Plasmodium knowlesi. Nature 2008; 455:799-803.

27. Kantele A, Marti H, Felger I, Müller D, Jokiranta ST. Monkey malaria in an European traveller returning from Malaysia. Emerg Infect Dis 2008; 14:1434-36.

28. Jongwutiwes S, Putaporntip C, Iwasatit T, Sata T, Kambara H. Naturally acquired Plasmodium knowlesi malaria in human, Thailand. Emerg Infect Dis 2004;10:2211-3.

29. Zhu HM, Li J, Zheng H. Human natural infection of Plasmodium knowlesi. Zhongguo Ji Sheng Chong Xue Yu Ji Sheng Chong Bing Za Zhi 2006; 24:70-1.

30. Ng OT, Eong OE, Chuan LC et al. Naturally acquired human Plasmodium knowlesi infection, Singapore. Emerg Infect Dis 2008; 14:814-6.

31. Bronner V, Divis PCS, Färnert A, Singh B. Swedish traveller with Plasmodium knowlesi malaria after visiting Malaysian Borneo. Malaria J 2009; 8:15:1-5. 